\title{
Reconstruction of electrons with the Gaussian-sum filter in the CMS tracker at LHC
}

\author{
W. Adam, R. Frühwirth \\ Institute for High-Energy Physics, Austrian Academy of Sciences, Vienna, Austria \\ A. Strandlie \\ CERN, Geneva, Switzerland \\ T. Todorov \\ IReS, Strasbourg, France
}

\begin{abstract}
The bremsstrahlung energy loss distribution of electrons propagating in matter is highly non Gaussian. Because the Kalman filter relies solely on Gaussian probability density functions, it might not be an optimal reconstruction algorithm for electron tracks. A Gaussian-sum filter (GSF) algorithm for electron track reconstruction in the CMS tracker has therefore been developed. The basic idea is to model the bremsstrahlung energy loss distribution by a Gaussian mixture rather than a single Gaussian. It is shown that the GSF is able to improve the momentum resolution of electrons compared to the standard Kalman filter. The momentum resolution and the quality of the estimated error are studied with various types of mixture models of the energy loss distribution.
\end{abstract}

\section{Introduction}

Modern track detectors based on semiconductor technologies contain larger amounts of material than gaseous detector types, partially due to the detector elements themselves and partially due to additional material required for on-sensor electronics, power, cooling, and mechanical support. A precise modelling of material effects in track reconstruction is therefore necessary to obtain the best estimates of the track parameters. Such material effects are particularly relevant for the reconstruction of electrons which, in addition to ionization energy loss and multiple Coulomb scattering, suffer from large energy losses due to bremsstrahlung.

A well-known model of the bremsstrahlung energy loss is due to Bethe and Heitler [1]. In this model, the probability density function (PDF), $f(z)$, of the energy loss of an electron is

$$
f(z)=\frac{[-\ln z]^{c-1}}{\Gamma(c)}
$$

where $c=t / \ln 2, t$ is the thickness of material traversed by the electron (in units of radiation length), and $z$ is the fraction of energy remaining after the material layer is traversed. The probability of a given fractional energy loss is assumed to be independent of the energy of the incoming particle. This PDF is shown in Fig. 1 for different thickness values.

The baseline for track reconstruction in the CMS tracker is the Kalman filter [2]. Throughout the filter tracks are described by a five-dimensional state vector, containing the information about the momentum, the direction and the position at some reference surface. The material effects are currently assumed to be concentrated in the active elements of the detector layers. In this context the optimal treatment of radiative energy loss is to correct the momentum with

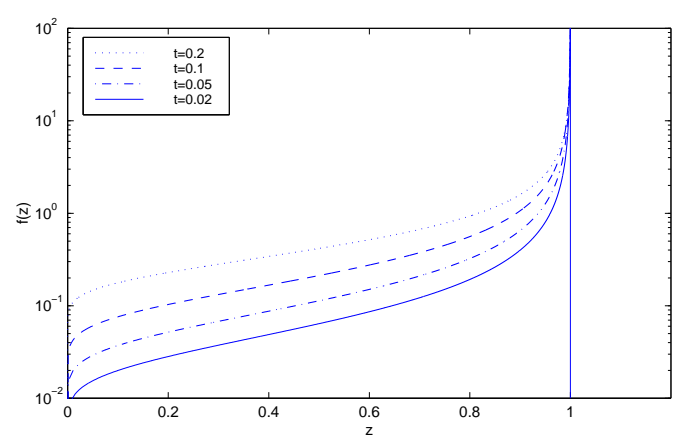

Figure 1: Probability density function $f(z)$ for different thickness values.

the mean value of energy loss and to increase the variance of the momentum by adding the variance of the energy loss distribution. This procedure should ensure unbiased estimates of the track parameters and of the associated uncertainties [3]. The Kalman filter is a linear least-squares estimator, and is proved to be optimal only when all probability densities encountered during the track reconstruction procedure are Gaussian. The implicit assumption of approximating the Bethe-Heitler distribution with a single Gaussian is quite crude. It is therefore plausible that a nonlinear estimator which takes the actual shape of the distribution into account can do better.

A non-linear generalization of the Kalman filter (KF), the Gaussian-sum filter (GSF) [4, 5], has therefore been implemented in the reconstruction software of the CMS tracker 6 . In the GSF the distributions of all state vectors are Gaussian mixtures, i.e. weighted sums of Gaussians instead of single Gaussians. The algorithm is therefore appropriate if the probability densities involved in track reconstruction can be adequately described by Gaussian mixtures. The basic 
idea of the present work is to approximate the BetheHeitler distribution as a Gaussian mixture rather than a single Gaussian, in which the different components of the mixture model different degrees of hardness of the bremsstrahlung in the layer under consideration. The resulting estimator resembles a set of Kalman filters running in parallel, where each Kalman filter corresponds to one of the components of the mixture describing the distribution of the state vector.

\section{Approximating the fractional energy loss distribution}

An important issue with the GSF reconstruction of electrons is to obtain a good Gaussian-mixture approximation of the Bethe-Heitler distribution. The parameters to be obtained are the weights, the mean values and the variances of each of the components in the approximating mixture. The parameters are determined by minimizing the following two distances:

$$
\begin{aligned}
D_{\mathrm{CDF}} & =\int_{-\infty}^{\infty}|F(z)-G(z)| d z, \\
D_{\mathrm{KL}} & =\int_{-\infty}^{\infty} \ln [f(z) / g(z)] f(z) d z,
\end{aligned}
$$

where $f(z)$ and $F(z)$ are the PDF and cumulative distribution function (CDF) of the model distribution and $g(z)$ and $G(z)$ are the PDF and CDF of the Gaussian mixture, respectively. The distance $D_{\mathrm{KL}}$ is the so-called Kullback-Leibler distance between the model distribution and the mixture. Hereafter, the mixtures obtained by minimizing $D_{\mathrm{CDF}}$ are called CDFmixtures, whereas the mixtures obtained by minimizing $D_{\mathrm{KL}}$ are called KL-mixtures. The minimizations have been done independently on a set of discrete values of $t$, ranging from 0.02 to 0.20 . Figures 2 and 3 show the resulting distances as a function of thickness for a varying number of components in the approximating mixture. In order to obtain mixtures for arbitrary values of the thickness, fifth-degree polynomials have been fitted to the parameters as a function of $t$. Due to the fast access to the parameters from the polynomials, the calculation of the mixture is done on the fly during reconstruction, using the effective thickness of a detector layer from the knowledge of the incident angle of inclination.

\section{Reducing the number of components}

The approximation of energy loss by a Gaussian mixture amounts to a convolution of this mixture with the current state, which in general is also composed of several Gaussian components. The strict application of the GSF algorithm therefore quickly leads to a
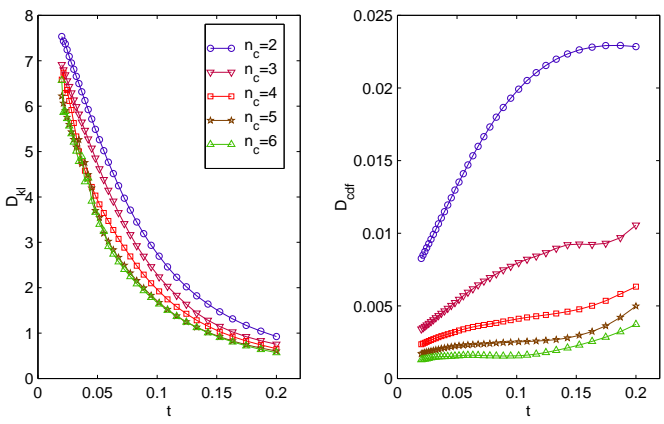

Figure 2: The distances $D_{\mathrm{KL}}$ and $D_{\mathrm{CDF}}$ as a function of the thickness $t$, for CDF-mixtures, with different numbers of components.
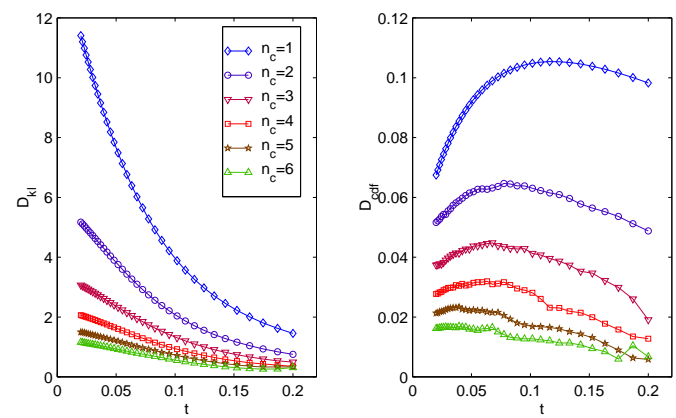

Figure 3: The distances $D_{\mathrm{KL}}$ and $D_{\mathrm{CDF}}$ as a function of the thickness $t$, for KL-mixtures, with different numbers of components.

prohibitively large number of components due to the combinatorics involved each time a layer of material is traversed.

In a realistic implementation of the GSF the number of components must repeatedly be reduced to a predefined maximum. As little information as possible should be lost in this procedure. Two strategies have been tested:

1. Only the $N$ components with the largest weights are kept;

2. Components are merged into clusters, according to a given metric.

The first option has the advantage of being computationally light, but it turns out to be inferior. Even the first two moments of the estimated parameters are not described correctly.

In the second approach, the component with the largest weight is merged with the one closest to it, and this procedure is repeated until the required number of components is reached. The results below have been obtained by using the Kullback-Leibler distance - defined in Equation (3) - as a measure of distance. 


\section{Results from simulated tracks in the CMS tracker}

First, results from the reconstruction of data originating from a simplified simulation are shown. In this simulation multiple scattering and ionization energy loss are turned off, all the material is concentrated on the detector units, and the exact amount of material used in the simulation is known by the reconstruction program. Single electron tracks with $p_{T}=10 \mathrm{GeV} / c$ have been simulated for absolute values of $\eta$ less than 1.0 . Reconstructed hits have been collected using the knowledge of the associated simulated hits, so no pattern recognition has been involved. The following results all refer to the quantity $q / p$ (charge over absolute value of the momentum) recorded at the point of closest approach to the vertex in the transverse plane - the transverse impact point (TIP) - after a fit going from the outside towards the inside of the tracker. Figure 4 shows an example of the estimated $q / p$ for one single track, both for the KF and for the GSF.

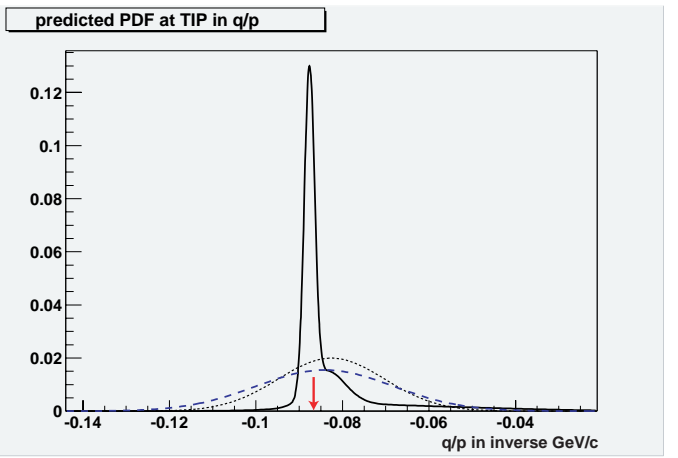

Figure 4: Estimated $q / p$ of one single track for the GSF (solid), the KF (dashed) and the combined GSF state (dotted). The combined GSF state refers to the first and the second moments of the GSF estimate, here visualized as a single Gaussian. The arrow denotes the true value of $q / p$. It can be seen that the estimated PDF of the GSF is a non-Gaussian function.

Figures 5 and 6 show probability distributions for the estimated $q / p$ of the KF and the GSF with a varying maximum number of components kept during the reconstruction. Given the estimated PDF (a single Gaussian for the KF, a Gaussian mixture for the GSF), each entry in the histogram amounts to the integral from $-\infty$ to the true value of $q / p$. If the estimated PDF is a correct description of the real distribution of the parameter, the corresponding histogram should be flat.

The deviation from flatness can be quantified by the $\chi^{2}$ of the difference between the probability distributions of $q / p$ and the flat distribution. This $\chi^{2}$ per bin is shown in Fig. 7 for a set of different mixtures as a function of the maximum number of components kept.

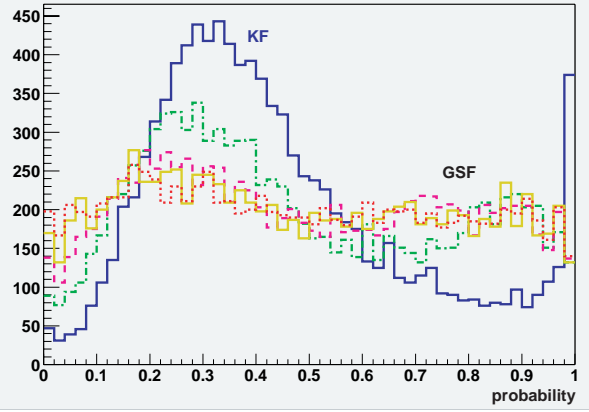

Figure 5: Probability distribution for the estimated $q / p$ for the KF (solid) and the GSF with a maximum of six (dashed-dotted), twelve (dashed), 18 (solid) and 36 (dotted) components kept during reconstruction. In this case the same six-component CDF-mixture has been used both in the simulation of the disturbance of the momentum in a detector unit and in reconstruction. Keeping 36 components yields estimates quite close to the correct distribution of the parameter.

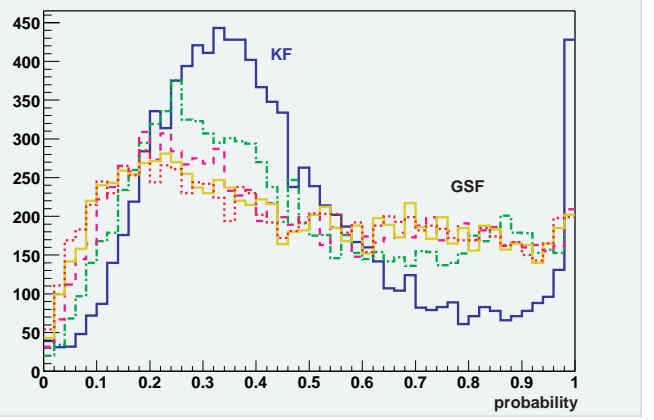

Figure 6: Probability distribution for the estimated $q / p$ for the KF (solid) and the GSF with a maximum of six (dashed-dotted), twelve (dashed), 18 (solid) and 36 (dotted) components kept during reconstruction. The same six-component mixture as the one described in the caption of Fig. 5 has been used in reconstruction, but the simulation of the disturbance of the momentum in a detector unit has been done by sampling from the Bethe-Heitler distribution. The distributions for the GSF are seen to be less flat than those shown in Fig. 5

The CDF-mixtures are superior to the KL-mixtures concerning the quality of the estimated $q / p$. The main trend seems to be related to the maximum number of components kept rather than the number of components in the mixture describing the energy loss, even though the mixtures with five and six components are best in the limit of keeping a large number of components.

Figure 8 shows the residuals of the estimated $q / p$ of the GSF and the KF with respect to the true value of 


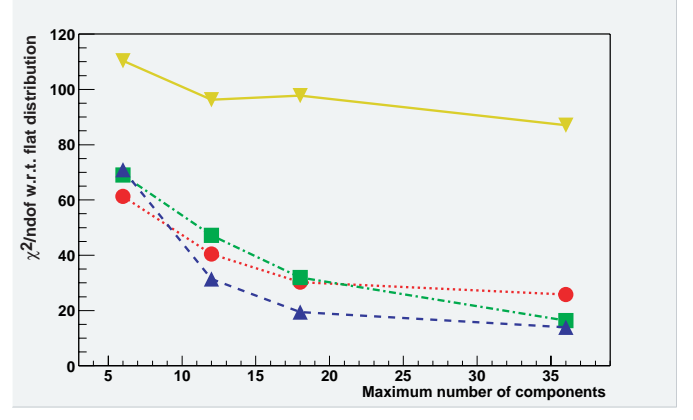

Figure 7: Values of $\chi^{2}$ per bin of the probability distribution for CDF-mixtures with four (circles), five (squares) and six (triangles pointing upwards) components, as well as a KL-mixture with six (triangles pointing downwards) components. The corresponding value for the $\mathrm{KF}$ is 146 .

the parameter. The estimated $q / p$ for the GSF is the mean value of the state vector mixture, and the mixture used for this specific plot is a CDF-mixture with six components. In order to quantify the difference between the GSF and the KF residuals, the full-width at half-maximum (FWHM) and the half-width of intervals covering $50 \%$ and $90 \%$ of the distribution have been considered. The covering intervals have been chosen to be symmetric about zero. The FWHM and the half-widths of the covering intervals are shown in Figs. 9] 10] and 11] The different flavours of the GSF in these figures are the same as those described in the caption of Fig. 7

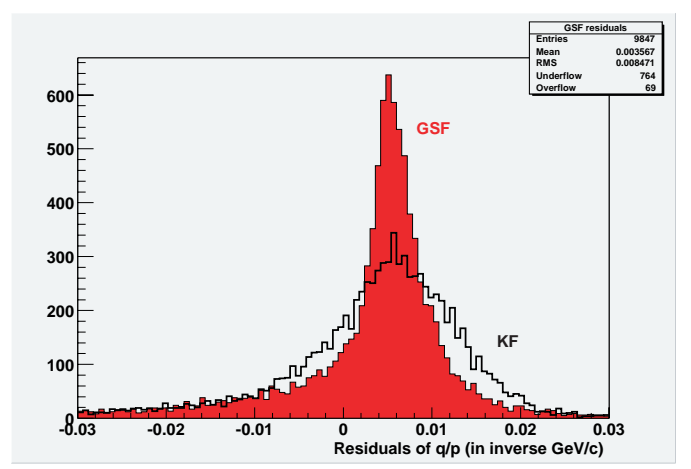

Figure 8: Residuals of estimated $q / p$ with respect to the true value of the parameter for the GSF and the KF at the transverse impact point. A maximum number of twelve components has been kept during reconstruction. Long tails extending outside the limits of the histogram exist both for the KF and for the GSF. These tails are due to hard radiation in the innermost layers of the tracker.

The GSF and the KF have also been run on tracks from a full simulation using the official CMS simula-

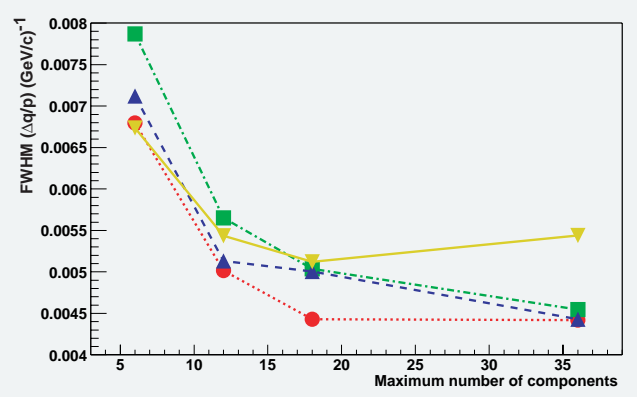

Figure 9: Full-width at half-maximum for the GSF as a function of the maximum number of components kept during reconstruction. The corresponding value of the $\mathrm{KF}$ is 0.013 .

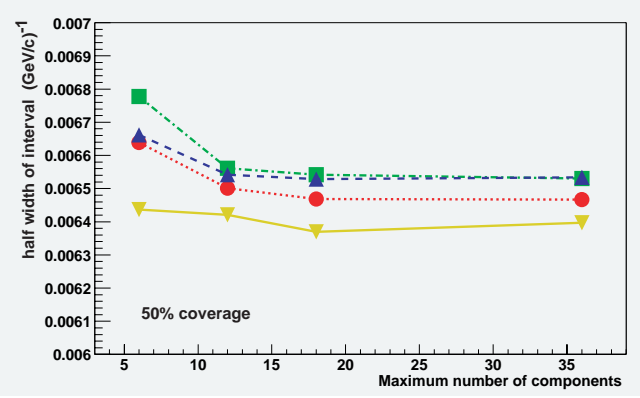

Figure 10: Half-widths of the symmetric intervals covering $50 \%$ of the distribution for the GSF as a function of the maximum number of components kept during reconstruction. The corresponding value of the $\mathrm{KF}$ is 0.0080 .

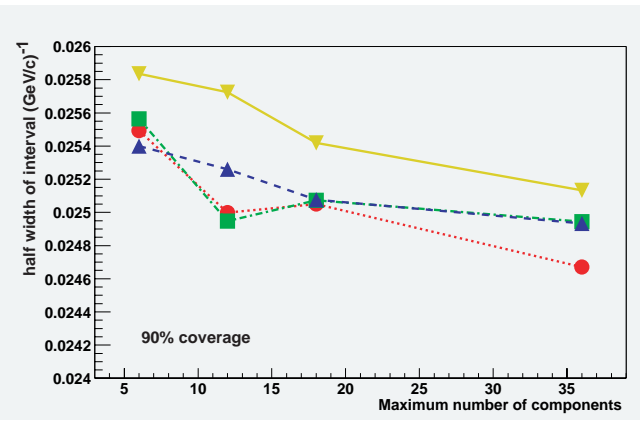

Figure 11: Half-widths of the symmetric intervals covering $90 \%$ of the distribution for the GSF as a function of the maximum number of components kept during reconstruction. The corresponding value of the $\mathrm{KF}$ is 0.0295 . 
tion program [7]. The $p_{T}$ and the $\eta$ range are the same as in the simplified simulation, but the amount and spatial distribution of the material are different. Probability distributions of the estimated $q / p$ for the GSF and the KF are shown in Fig. 12 The prob-

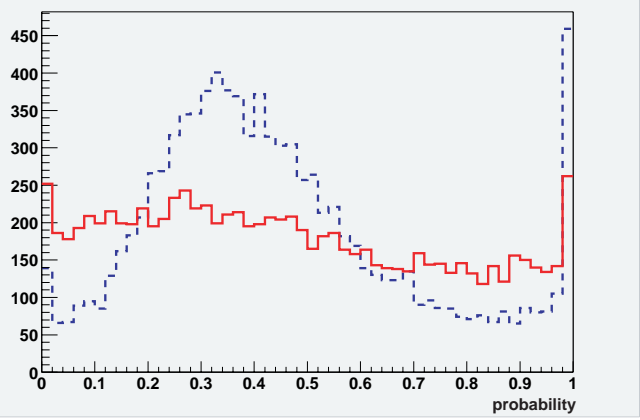

Figure 12: Probability distribution for the estimated $q / p$ for the KF (dashed) and the GSF (solid). The specific mixture used in the GSF is a CDF-mixture with six components. A maximum number of twelve components has been kept during reconstruction. The reconstruction algorithms have been run on tracks from a full simulation.

ability distribution of the GSF exhibits no large deviation from flatness, indicating that the estimated $\mathrm{PDF}$ of $q / p$ describes reasonably well the actual PDF of $q / p$. This observation is all the more remarkable since, with the full simulation, the energy loss is not generated by the simple Bethe-Heitler model, and neither the exact amount nor the exact location of the material are known to the GSF.

The corresponding residuals of the estimated $q / p$ with respect to the true value are shown in Figs. 13 and 14. The residuals shown in Fig. 14 have been obtained by including a vertex constraint in the fit. Such a constraint allows the momentum to be measured in the innermost part of the track and thus gives a handle on possible radiation in the first two layers. The result of including this constraint is a less skew distribution with the mode being moved closer towards zero, and the amount of tracks in the tails is also reduced. Even though the results from the full simulation qualitatively seem to confirm those from the simplified simulation, more studies are needed to understand the differences in detail.

\section{Conclusion}

The Gaussian-sum filter has been implemented in the CMS reconstruction program. It has been validated with electron tracks with a simplified simulation in which the energy loss distribution (Bethe-Heitler model), the exact amount of material and its exact

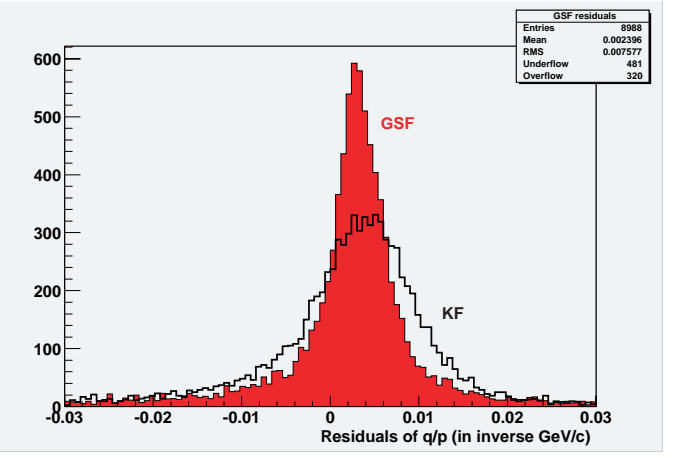

Figure 13: Residuals of the estimated $q / p$ with respect to the true value at the transverse impact point for the KF and the GSF. The reconstruction algorithms have been run on tracks from a full simulation.

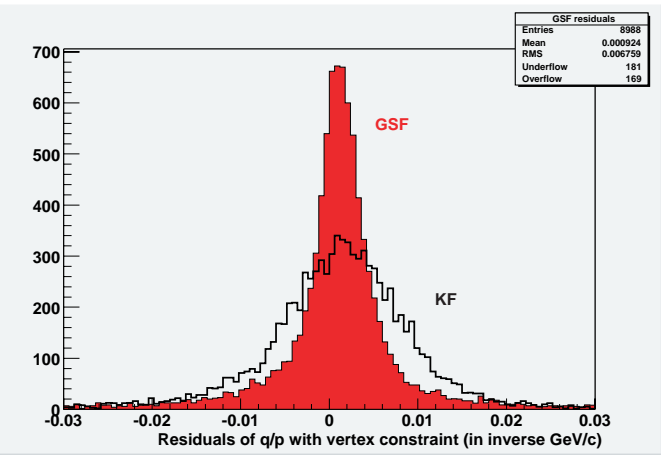

Figure 14: Residuals of the estimated $q / p$ with respect to the true value at the transverse impact point for the $\mathrm{KF}$ and the GSF. A vertex constraint has been included in the fits. The reconstruction algorithms have been run on tracks from a full simulation.

location are known to the reconstruction program. It has been shown that the quality of the momentum estimate depends mainly on the number of mixture components kept during reconstruction, and to some extent also on the number of components in the mixture approximation to the energy loss distribution. A comparison with the best linear unbiased estimator, the Kalman filter, shows a clear improvement of the momentum resolution. Remarkably, a similar improvement can be seen with electron tracks from the full simulation, although in this case neither the exact energy loss distribution nor the precise amount and location of material are known to the reconstruction program. More systematic studies with electrons from the full simulation are clearly needed, but it seems safe to conclude that in electron reconstruction the Gaussian-sum filter yields a substantial gain in precision as compared to the Kalman filter. 


\section{References}

[1] H. Bethe and W. Heitler, Proc. R. Soc. London A 146 (1934) 83.

[2] R. Frühwirth, Nucl. Instrum. and Methods A 262 (1987) 444.

[3] D. Stampfer, M. Regler and R. Frühwirth, Comp.
Phys. Comm. 79 (1994) 157.

[4] R. Frühwirth, Comp. Phys. Comm. 100 (1997) 1.

[5] R. Frühwirth and S. Frühwirth-Schnatter, Comp. Phys. Comm. 110 (1998) 80.

[6] http://cmsdoc.cern.ch/orca/

[7] http://cmsdoc.cern.ch/cmsim/cmsim.html 